\title{
Elastic measurements on macroscopic three-dimensional pentamode metamaterials
}

\author{
Robert Schittny ${ }^{1}$, Tiemo Bückmann ${ }^{1}$, Muamer Kadic ${ }^{1}$, and Martin Wegener ${ }^{1,2}$ \\ ${ }^{1}$ Institute of Applied Physics, Karlsruhe Institute of Technology (KIT), \\ 76128 Karlsruhe, Germany \\ ${ }^{2}$ Institute of Nanotechnology, Karlsruhe Institute of Technology (KIT), \\ 76128 Karlsruhe, Germany
}

Pentamode metamaterials approximate tailorable artificial liquids. Recently, microscopic versions of these intricate three-dimensional structures have been fabricated, but direct experimental characterization has not been possible yet. Here, using three-dimensional printing, we fabricate macroscopic polymer-based samples with many different combinations of the small connection diameter $d$ and the lattice constant $a$. Direct measurements of the static shear modulus and the Young's modulus reveal that both scale approximately according to $(d / a)^{3}$, in good agreement with continuum-mechanics calculations. For the smallest accessible values of $d / a \approx 1.5 \%$, we find derived ratios of bulk modulus $B$ to shear modulus $G$ of $B / G \approx 1000$.

Pentamode elastic metamaterials [1-3] are rationally designed artificial crystals allowing for obtaining an effective metamaterial shear modulus $G$ which can be orders of magnitude smaller than the effective bulk modulus $B$. Intuitively, this means that it is much easier to change the shape of such a metamaterial (while fixing its volume) than it is to change its volume (while fixing its shape). In this sense, pentamode metamaterials approximate the elastic properties of liquids. In contrast to liquids, however, it is straightforward to envision stable and intentionally spatially inhomogeneous as well as anisotropic [4,5] pentamode metamaterial architectures. Normal liquids would simply flow away or intermix. Hence, such metamaterial structures are promising for experimentally translating the concepts of transformation optics [6] 8] to elastostatics and elastodynamics [9-12]. For example, one could design and realize elastostatic cloaks, which would make some hard interior unfeelable from the outside.

Pentamode metamaterials were already suggested theoretically in 1995 [1, but have been realized experimentally only in 2012 [2]. However, the microscopic samples fabricated there [2] by using dip-in direct laser writing could not be characterized directly. In this Letter, taking advantage of the scalability of continuum mechanics, we fabricate 
much larger macroscopic versions of pentamode metamaterials with lattice constants of $a \approx 1 \mathrm{~cm}$. This step enables direct measurement of the elastic properties as a function of the critical geometrical parameter, i.e., the diameter of the small connections within the pentamode unit cell.

Fig. 1 shows a gallery of selected photographs of a number of pentamode metamaterial structures that we have fabricated using a three-dimensional printer (Objet30 by formerly Objet, now Stratasys, USA). For the printing process of the geometrically complex pentamode metamaterials, a support material is needed that holds all overhanging parts and can be removed later. We choose Stratasys' proprietary "FullCure850 VeroGray" polymer as the model material and pure "FullCure705 Support" as support, which can be etched out after printing in a bath of $\mathrm{NaOH}$ base. The default support material (a fine-grained mixture of "FullCure850 VeroGray" and "FullCure705 Support") has proven to be impossible to remove from the delicate pentamode structures. The pentamode metamaterials are composed of double-cone elements, the tips of which touch each other at a set of fictitious points forming a diamond lattice [1,2]. We have previously shown by numerical modeling [2, 3] that the diameter $d$ of the resulting touching region compared to the lattice constant $a$ of the face-centered-cubic (fcc) translational lattice (see Fig. 1 for the geometrical dimensions) is the most important parameter in terms of influence on the effective properties. Precisely, the ratio of bulk to shear modulus scales like $B / G \propto(a / d)^{2}$. This scaling results from the approximate dependencies $B \propto d / a$ and $G \propto(d / a)^{3}$. In contrast, we have shown that the diameter $D$ of the thick end of the cones is less important [2]. Intuitively, as so often in life, "the weakest link in a chain determines its properties".

The relevant $B / G$ ratio is directly connected to the effective metamaterial Poisson's ratio $\nu$ [13], which therefore could in principle be used as a measure for characterization. For $B / G \rightarrow \infty$, one gets $\nu \approx 0.5\left(1-(B / G)^{-1}\right) \rightarrow 0.5$ [2]. This means that very large $B / G$ ratios would have to be determined from minute deviations of $\nu$ from its upper bound of 0.5 , requiring measurements of $\nu$ with at least three significant digits. Such precision measurements appear neither attractive nor in reach to us. Thus, we rather measure the shear modulus via its definition, i.e., we impose a shear displacement and measure the shear force using a force cell ("K3D60N 10 N" from ME-Meßsysteme, Germany). Together with the known area the force is acting upon, the shear modulus follows directly and quantitatively.

A photograph of the corresponding computer-controlled measurement setup is shown in Fig. 2. To obtain well-defined and reproducible boundary conditions, we directly and monolithically print each of the to-be-characterized pentamode metamaterial samples with rigid plates on the top and bottom which then are fixed to the metal stamps of the measurement setup. A representative force versus strain measurement within the linear regime is depicted in Fig. 3(a). As usual for viscoelastic materials, one gets a certain hysteresis when linearly ramping up and down the shear strain over a total time period of about $100 \mathrm{~s}$. The purely elastic component of the shear modulus is obtained from the fitted slope (see straight line in Fig. 3(a)). Repeating measurements like that for many different samples with different values of $d$ and $a$ and for both the $x$ - and the $y$-direction leads to the data points summarized in Fig. 4, which uses a double-logarithmic representation of the moduli in dependence on $d / a$. Apart from some scatter, the measured 


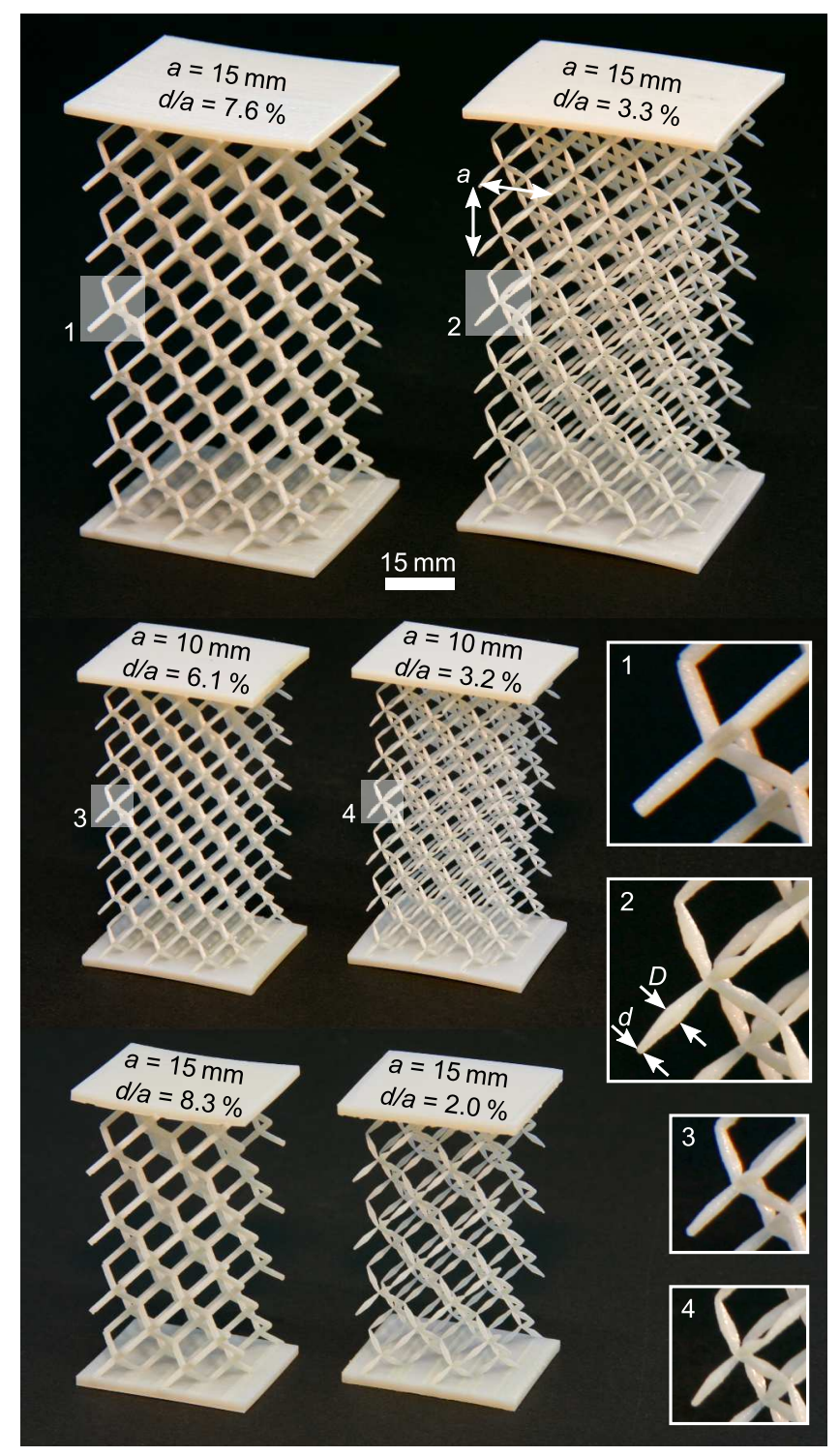

Figure 1: Gallery of photographs of selected macroscopic polymer-based pentamode metamaterial samples fabricated using three-dimensional printing. The insets show magnified parts of the samples as indicated by the white overlays. 


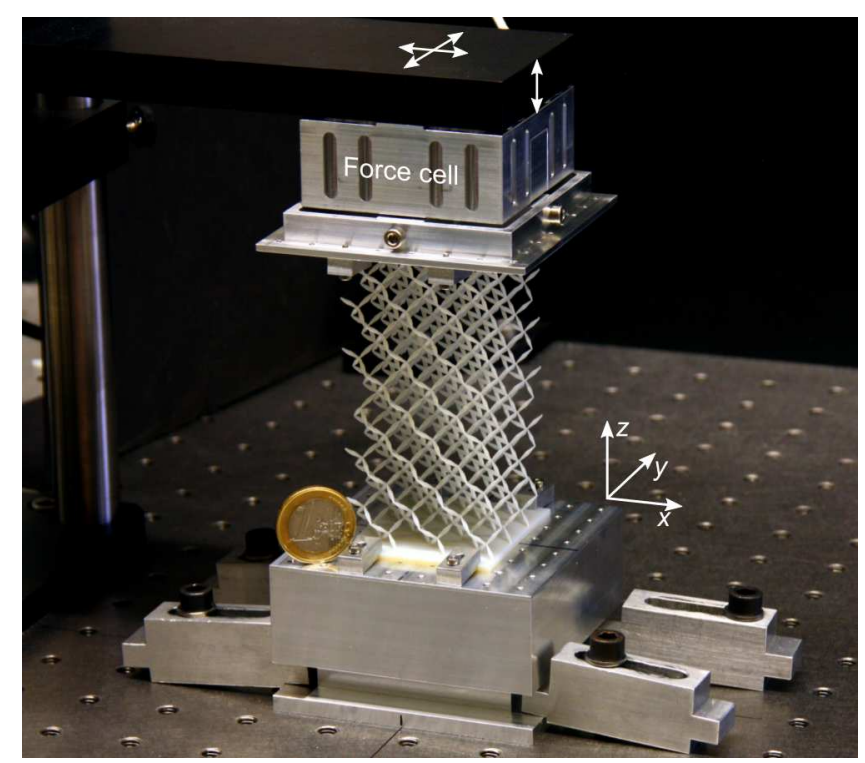

Figure 2: Photograph of the experimental setup including a force cell, allowing for direct shear and Young's modulus measurements. The bottom and top metal stamps of this setup are rigidly attached to polymer plates that have been fabricated monolithically in the three-dimensional polymer-printing process.

data points (colored circles) roughly follow the anticipated scaling $G \propto(d / a)^{3}$ (see black straight line). We note that the geometrical parameters $d, D$, and $a$ have directly been determined from an analysis of photographs of the actually fabricated and investigated samples. The measured geometrical parameters for $d$ and $D$ show deviations from the nominal ones (that are fed into the $3 \mathrm{D}$ printer) by approximately $10 \%$. The horizontal error bars in Fig. 4 correspond to the scatter of the determined $d$ values in each sample. The measurements of $G$ (colored circles) are compared with numerical calculations for the shear modulus (black circles). For details on the numerical calculations, we refer the reader to Ref. 2. We take the identical geometrical parameters and the identical number of extended pentamode unit cells as in the present experiments. Using a Young's modulus of $1.4 \mathrm{GPa}$ and a Poisson's ratio of 0.4 (the latter being not critical at all [2]) for the constituent polymer material, we obtain good agreement with the experimental data (compare black and colored circles in Fig. 44). Here, the Young's modulus of $1.4 \mathrm{GPa}$ has been the only adjustable parameter. According to the underlying continuum-mechanics equations, it only scales all effective metamaterial moduli by a common factor, but it strictly neither influences the exponent versus $d / a$ nor any ratio between effective metamaterial moduli. We have also performed different independent Young's modulus measurements on bulk pieces of the printed constituent polymer "FullCure850 VeroGray", leading to values of $0.7-2.0 \mathrm{GPa}$. The value quoted by the provider is $2-3 \mathrm{GPa}$. The value of $1.4 \mathrm{GPa}$ chosen above lies within this significant scatter.

Next, one would ideally like to measure the effective metamaterial bulk modulus $B$ directly as well. To do so, in principle, one needs to simultaneously and equally push 

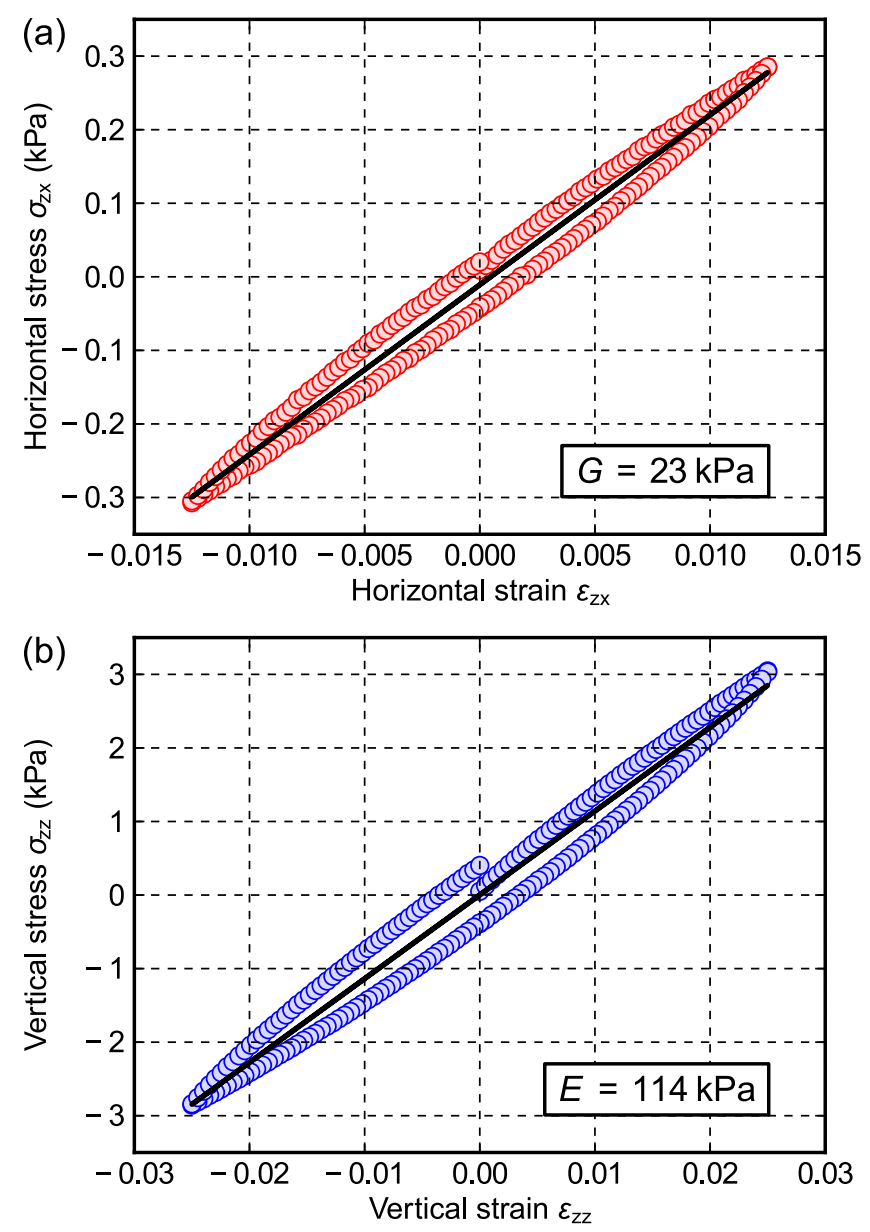

Figure 3: (a) Example of an individual shear-modulus measurement (red circles). (b) Example of one Young's-modulus measurement (blue circles). The moduli are obtained from the slopes of the fitted straight lines. The geometrical parameters (see Fig. 11) of the sample composed of $2 \times 2 \times 4$ extended unit cells are: $d=0.71 \mathrm{~mm}, D=1.32 \mathrm{~mm}$, and $a=15 \mathrm{~mm}$. 
from all six sides of the cuboid samples under sliding boundary conditions and measure the corresponding displacements and forces. Such a measurement appears challenging to us. We thus rather follow a different, somewhat more indirect but readily feasible approach: We also measure the Young's modulus, again compare to theory, and then, assuming good overall agreement, use theory to derive the bulk modulus. In more detail, it is simple to show that the effective metamaterial Young's modulus $E$ should follow $E \approx 3 G$ in the limit of $B / G \rightarrow \infty$. To measure the Young's modulus, we again use the setup shown in Fig. 2 but impose a displacement along the vertical direction (i.e., a given strain) and measure the resulting vertical force component with the force cell. From these, the Young's modulus follows according to its definition. To allow for direct comparison with the shear data, we use the same samples as for the shear measurements. Due to the mentioned mounting plates, however, the samples experience fixed boundary conditions at the stamps along both horizontal directions. In an ideal Young's modulus measurement, the boundary conditions should rather be sliding. Thus, we use fixed boundary conditions in our numerical modeling as well. An example of a measured stress-strain relation is depicted in Fig. 3(b). The Young's modulus results from the slope of the indicated straight line. Repeating this experiment for all samples leads to the set of colored triangles exhibited in Fig. 4, The corresponding calculated values are also shown there as black triangles. Obviously, without any further adjustable parameters, experiment and theory agree nicely in regard to the Young's modulus as well. In the double-logarithmic representation, the Young's modulus data points are parallel to the shear modulus data points. This means that the Young's modulus scales with the same exponent of 3 versus $d / a$. Based on the good overall agreement between experiment and theory in regard to $G$ and $E$ versus $d / a$, we consider calculated values of $B$ as reliable and trustworthy. Since edge effects would dominate in this calculation for the sample sizes used here, we rather consider a fictitious infinitely extended crystal. We assume that all extended fcc unit cells therein are compressed equally in all three cubic directions upon imposing a pressure from these three directions simultaneously. Technically, choosing a coordinate system with a fixed center of gravity, this translates into "anti-symmetric" boundary conditions for the normal component of the displacement vector on opposing faces of the extended fcc unit cell. The calculated data points for $B$ are shown as black squares in Fig. 4. At the smallest accessible ratios of $d / a \approx 1.5 \%$ on the left-hand side, the $B / G$ ratio is as large as 1000 (see double-arrow). Even at the largest ratios of $d / a \approx 9 \%$ on the right-hand side (where the structure's double-cones essentially become straight rods), the $B / G$ ratio is still on the order of 100 (see double-arrow).

Let us finish by noting that our discussion above has tacitly assumed that the effective metamaterial properties are isotropic (otherwise $B$ and $G$ would turn from scalars into tensors), while the pentamode unit cell only guarantees cubic crystal symmetry. In general, for a cubic crystal, Poisson's ratio is not isotropic and can even exhibit rather complex and pronounced direction dependencies [14]. However, it has been shown [14] that a Poisson's ratio approaching 0.5 from below for pushing along the principal axes of a cubic crystal leads to an isotropic Poisson's ratio $\nu \rightarrow 0.5$. This means that our metamaterial structures approximate isotropic liquids. Anisotropic versions of three-dimensional [4] and two-dimensional [5] pentamode metamaterials have also been discussed theoretically (strictly speaking, there is no such thing as a two-dimensional isotropic pentamode meta- 


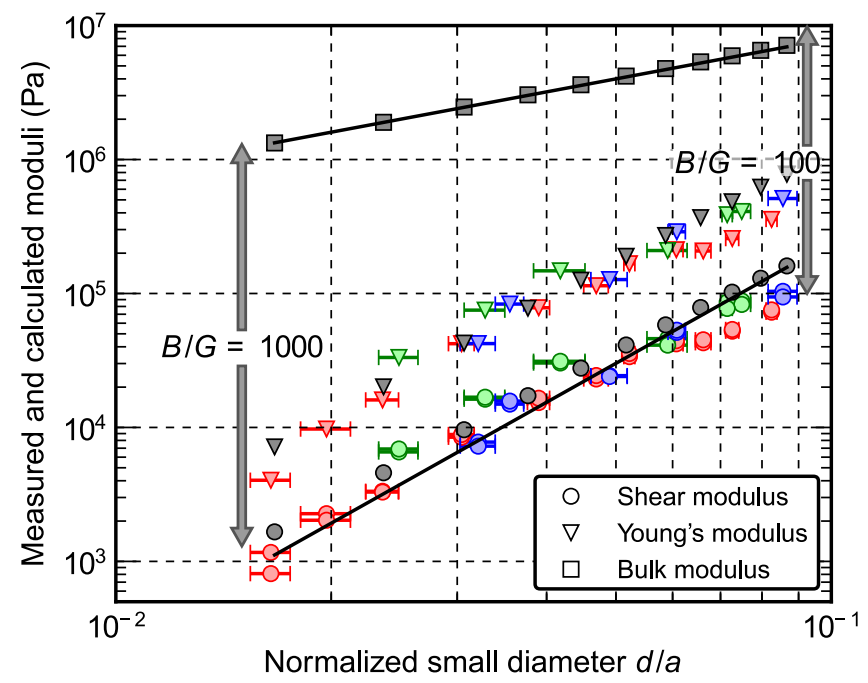

Figure 4: Elastic moduli (real parts) versus the ratio $d /$ a of the diameter of the small double-cone connections $d$ and the pentamode fcc lattice constant $a$ in doublelogarithmic representation. Shown are the shear modulus $G$ as circles, the Young's modulus $E$ as triangles, and the bulk modulus $B$ as squares. The colored symbols correspond to experimental data (also see Fig. 3), the black symbols to numerically calculated values for the geometrical parameters taken from the experiment. The sample parameters are $a=1.5 \mathrm{~cm}$ and $2 \times 2 \times 4$ extended unit cells (red), $a=1.5 \mathrm{~cm}$ and $3 \times 3 \times 6$ extended unit cells (green), and $a=1.0 \mathrm{~cm}$ and $3 \times 3 \times 6$ extended unit cells (blue). The only other geometrical parameter, the diameter of the thick end of the double cones is fixed to $D / a=0.087$ for all samples. The two black solid straight lines are guides to the eye (not fits) and correspond to scalings of $G \propto(d / a)^{3}$ and $B \propto(d / a)^{1}$, respectively. The two vertical double-arrows on the left and on the right highlight the approximate $B / G$ ratio for the smallest and the largest investigated ratios of $d / a$. A ratio of $B / G=1000$ means that the pentamode metamaterial approximates well the properties of an isotropic liquid. 
material, it is rather a bimode metamaterial). It is presently not clear though how to fully characterize the complex elasticity tensor of such structures with non-cubic crystal symmetry in direct static experiments.

In conclusion, we have measured the effective shear modulus and the effective Young's modulus on a large set of different macroscopic three-dimensional polymer-based pentamode metamaterials. The good agreement of experiment and theory allows us to extract the ratios of bulk to shear modulus. We find ratios as large as $B / G \approx 1000$, which were previously predicted only purely theoretically [2]. This finding raises hopes that threedimensional elastic cloaks are indeed in reach experimentally. For example, theoretical work on pentamode-based circular cloaks [15] has shown good cloaking for ratios of $B / G$ of 100 and even excellent cloaking for ratios of 1000. A bulk of theoretical work which has assumed that ideal pentamode materials are available [15] is now backed up by an experimental characterization of three-dimensional pentamode elastic metamaterials in the static limit.

We thank Prof. Manfred Wilhelm (KIT) for rheological measurements on the bulk printing material. We acknowledge support from the DFG-Center for Functional Nanostructures (CFN) and the Karlsruhe School of Optics \& Photonics (KSOP).

\section{References}

[1] G. W. Milton and A. V. Cherkaev, J. Eng. Mater. Technol. 117, 483 (1995).

[2] M. Kadic, T. Bückmann, N. Stenger, M. Thiel, and M. Wegener, Appl. Phys. Lett. 100, 191901 (2012).

[3] A. Martin, M. Kadic, R. Schittny, T. Bückmann, and M. Wegener, Phys. Rev. B 86, 155116 (2012).

[4] M. Kadic, R. Schittny, T. Bückmann, and M. Wegener, New J. Phys. 15, 023029 (2013).

[5] C. N. Layman, C. J. Naify, T. P. Martin, D. C. Calvo, and G. J. Orris, Phys. Rev. Lett. 111, 024302 (2013).

[6] J. B. Pendry, D. Schurig, and D. R. Smith, Science 312, 1780 (2006).

[7] D. Schurig, J. J. Mock, B. J. Justice, S. A. Cummer, J. B. Pendry, A. F. Starr, and D. R. Smith, Science 314, 977 (2006).

[8] U. Leonhardt, Geometry and Light: The Science of Invisibility (Dover, Mineola, 2010).

[9] G. W. Milton, M. Briane, and J. R. Willis, New J. Phys. 8, 248 (2006).

[10] A. N. Norris, Proc. R. Soc. London, Ser. A 464, 2411 (2008).

[11] M. Brun, S. Guenneau, and A. B. Movchan, Appl. Phys. Lett. 94, 061903 (2009).

[12] A. N. Norris and A. L. Shuvalov, Wave Motion 48, 525 (2011). 
[13] P. L. Gould, Introduction to Linear Elasticity (Springer-Verlag, 1994).

[14] K. W. Wojciechowski, Computational Methods in Science and Technology 11, 73 (2005).

[15] Y. Urzhumov, F. Ghezzo, J. Hunt, and D. R. Smith, New J. Phys. 12, 073014 (2010).

[16] A. N. Norris, J. Acoust. Soc. Am. 125, 839 (2009).

[17] S. C. Scandrett, J. E. Boisvert, and T. R. Howarth, J. Acoust. Soc. Am. 127, 2856 (2010).

[18] J. Cipolla, N. Gokhale, A. N. Norris, and A. Nagy, J. Acoust. Soc. Am. 130, 2332 (2011).

[19] N. H. Gokhale, J. L. Cipolla, and A. N. Norris, J. Acoust. Soc. Am. 132, 2932 (2012). 